\title{
Interações planta medicinal x medicamento convencional no tratamento da hipertensão arterial
}

\author{
Medicinal plant versus conventional medicine interaction \\ in arterial hypertension treatment
}

Recebido em: 29/09/2016

Aceito em: 10/03/2017
Júlia Beatriz Pereira SOUZAㅜㄹ Fábia Jéssica Batista ATALIBA²; Danielly Albuquerque da COSTA $^{3}$; Andrezza Duarte FARIAS ${ }^{1}$

${ }^{1}$ Universidade Federal de Campina Grande, Centro de Educação e Saúde, Sítio Olho D'água da Bica S/N, Centro, CEP: 58175-000. Cuité, PB, Brasil. ${ }^{2} I P O G$ - Instituto de Pós-Graduação e Graduação. Av. Monsenhor Walfredo Leal, 503, Centro, CEP: 58020-540. João Pessoa, PB, Brasil. ${ }^{3}$ Universidade Federal da Paraíba, Centro de Ciências da Saúde, Cidade Universitária, S/N, Castelo Branco, CEP: 58051-900.João Pessoa, PB, Brasil.E-mail: juliabtriz@gmail.com

\section{ABSTRACT}

Hypertension, among chronic diseases, appears as health problem with one of the greatest magnitude, at present is one of the main causes of cardiovascular morbidity and mortality in Brazil. The treatment consists in the use of synthetic drugs and medicinal plants, and this association is getting even more frequent, increasing the risks of interactions. This work is aimed to describe, through a literature review, the main interactions between herbal and synthetic drugs used in the therapy of hypertension and discuss the implications of these products concomitant use. A narrative literature review was carried out in papers published in the period 2006-2016 addressing drug interactions with medicinal plants from the ethnopharmacological survey, followed by studies of antihypertensive activity confirmation. The plants recognized for the hypertension treatment were Allium sativum, Alpinia zerumbet, Citrus sp., Lippia alba, Passiflora sp., Cymbopogon citratus, Sechium edule. Possible interactions of these plants with synthetic drugs to treat hypertension were evaluated. It was noted that the main interactions involved in the use of herbal associated with the use of medications are a pharmacodynamic type. Thus it is necessary to be cautious when associating the use of plants with synthetic drugs.

Keywords: phytotherapy; drug interactions; hypertension.

\section{RESUMO}

A hipertensão arterial sistêmica, dentre as doenças crônicas, apresenta-se como um dos problemas de saúde de maior magnitude na atualidade, sendo uma das principais causas de morbidade e mortalidade cardiovascular no Brasil. Seu tratamento consiste na utilização de medicamentos convencionais e plantas medicinais, sendo essa associação cada vez mais frequente, aumentando os riscos de ocorrência de interações. Este trabalho teve como objetivo descrever, mediante revisão de literatura, as principais interações entre plantas medicinais e medicamentos sintéticos utilizados na terapia da hipertensão arterial e discutir as implicações do uso concomitante desses produtos. Foi realizada uma revisão da literatura em trabalhos publicados no período de 2006 a 2016, abordando interações de medicamentos com plantas medicinais a partir de levantamento entofarmacológico, seguido de estudos de comprovação da atividade anti-hipertensiva. As plantas reconhecidas para o tratamento de hipertensão foram Allium sativum, Alpinia zerumbet, Citrus sp., Lippia alba, Passiflora sp., Cymbopogon citratus, Sechium edule. Foram avaliadas as possíveis interações dessas plantas com os medicamentos convencionais para tratar hipertensão. Foi observado que as principais interações envolvidas no uso concomitante entre plantas medicinais e fármacos são do tipo farmacodinâmicas. Dessa forma se faz necessário ter cautela ao associar o uso de plantas aos medicamentos.

Palavras-chave: fitoterapia; interação de medicamentos; hipertensão arterial. 


\section{INTRODUÇÃO}

As doenças crônicas ocupam o quadro de maior causa de morbidade e mortalidade no país. Geralmente sua progressão é lenta, e permanecem por longos períodos, desenvolvendo efeitos a longo prazo, raramente previsíveis $(1,2)$. A hipertensão arterial sistêmica é uma comorbidade que não possui uma causa definida, sendo caracterizada pela permanência de elevados níveis tensoriais, associados a mudanças metabólicas, hormonais e a fenômenos tróficos, como a hipertrofia cardíaca vascular (3). Existem vários fatores de risco que predispõem seu desenvolvimento, entre eles: o sedentarismo, excesso de peso, tabagismo e alimentação inadequada $(4,5)$.

Plantas têm sido usadas terapeuticamente por milhares de anos e continuam a ser a principal modalidade de tratamento para uma grande parcela da população mundial. Além disso, o uso da fitoterapia está aumentando nos países ocidentais como tratamento complementar $\mathrm{e}$, às vezes, alternativo, em conjunto com a medicina convencional (6). Em todo o mundo, os medicamentos à base de plantas desempenham um papel importante nos programas de cuidados de saúde (7).

Atualmente, milhões de pessoas utilizam plantas juntamente com medicamentos prescritos e não prescritos (6-8). Apesar de ser considerado natural e seguro, muitas dessas plantas podem interagir com outros medicamentos, causando efeitos colaterais potencialmente perigosos e / ou redução dos benefícios obtidos com o tratamento convencional (8).

A atividade farmacológica obtida através de espécies vegetais resulta de uma ou de um conjunto de substâncias ativas de grande complexidade que pode modular e modificar os efeitos de princípio(s) ativo(s). Além disso, plantas podem apresentar constituintes em concentrações diversas que interagem com muitas outras substâncias resultantes do metabolismo primário ou secundário da planta, diferente dos medicamentos sintéticos $(7,9)$. Deve ser entendido que as preparações à base de plantas contêm vários fitoquímicos ativos em proporções variáveis que podem, como qualquer outra substância farmacológica ativa, alterar os sistemas enzimáticos, transportadores e/ou processos fisiológicos (10).

Uma preocupação é em relação ao uso de fitoterapia sem conhecimento médico, pois, quando usada em conjunto com outros medicamentos podem acarretar interações e efeitos tóxicos não esperados pelo médico ou provocar hepatotoxicidade $(11,12)$. As interações entre um princípio ativo e os componentes químicos que estão contidos nas plantas medicinais possuem potencial para causar alterações nas concentrações plasmáticas dos medicamentos, comprometendo, dessa forma, sua efetividade e segurança. De acordo com o mecanismo de ação, as interações podem ser classificadas em farmacocinéticas e farmacodinâmicas (13).

Investigações clínicas e experimentais têm centrado sobre os efeitos e mecanismos de ação de plantas medicinais no tratamento de doenças cardiovasculares (14). Segundo Saad ee cols (2016), as plantas podem ser utilizadas para o tratamento da hipertensão arterial, de maneira isolada na hipertensão leve, ou em conjunto com anti-hipertensivos sintéticos nas moderadas e graves (15).

Em virtude das plantas medicinais representarem uma alternativa terapêutica largamente utilizada, principalmente por portadores de doenças crônicas, faz-se necessário conhecer as plantas que interagem com os medicamentos anti-hipertensivos, no intuito de que essa informação seja divulgada e utilizada para minimizar ou prevenir riscos causados por essa associação na população em geral, uma vez que há poucos estudos e uma carência de informação sobre a referida temáti

\section{MÉTODOS}

Trata-se de uma revisão da literatura, desenvolvida a partir da análise e descrição de uma parte do conhecimento disponível em artigos científicos e livros que abordam o tema em questão, em busca de respostas a uma pergunta específica (16). Esta abordagem permite ao pesquisador compreender fenômenos, principalmente quando o problema de pesquisa abrange dados muito dispersos (17).

A pesquisa foi realizada por meio das bases de dados SciELO, LILACS, MEDLINE, PubMed, BVS e Portal CAPES, a partir de trabalhos publicados entre 2006 e 2016, utilizando os descritores Hipertensão arterial, Plantas medicinais na hipertensão, Interação de plantas com medicamentos e Fitoterapia.

Inicialmente foram selecionados 87 artigos incluindo artigos originais, artigos de revisão e editoriais, escritos na língua portuguesa ou inglesa, além de livros, que abordassem informações pertinentes a temática em estudo. Destes, foram utilizados 37 artigos, após exclusão de todos os trabalhos que não contemplavam informações relevantes ao tema pesquisado. 


\section{RESULTADOS E DISCUSSÃO}

A partir do levantamento etnofarmacológico, foram reconhecidas 47 espécies, pertencentes a 23 famílias de plantas utilizadas para o tratamento de hipertensão arterial. Dessas, foram eleitas 7 espécies pertencentes a 6 famílias (Quadro 1), por serem as mais citadas e apresentarem estudos científicos comprovando atividade anti-hipertensiva.

Quadro 1. Plantas medicinais com atividade anti-hipertensiva.

\begin{tabular}{|c|c|c|c|}
\hline Família, Nome Científico, Nome Vulgar & Parte Utilizada & Ação & Referência \\
\hline Alliaceae, Allium sativum L., Alho & Bulbo composto & $\begin{array}{l}\text { Coadjuvante no tratamento da hipertensão; } \\
\text { tratamento da hipertensão arterial leve }\end{array}$ & (18) \\
\hline $\begin{array}{c}\text { Zingiberaceae, Alpinia zerumbet (Pers.) B.L. } \\
\text { Burtt \& R.M. Sm., Colônia }\end{array}$ & Folha & Redução da pressão arterial média & (19) \\
\hline Passifloraceae, Passiflora sp., Maracujá & Folha & $\begin{array}{l}0 \text { efeito antihipertensivo pode ser devido à } \\
\text { valorização do estado antioxidante }\end{array}$ & $(20,21)$ \\
\hline Rutaceae, Citrus sp., Laranja & Folha, fruto & Efeito hipotensor & $(22,23)$ \\
\hline $\begin{array}{c}\text { Verbenaceae, Lippia alba (Mill.) N.E.Br., Erva } \\
\text { cidreira, Lípia }\end{array}$ & Folha, flor & $\begin{array}{l}\text { Redução da pressão arterial por um efeito direto } \\
\text { sobre o músculo liso vascular levando a vasodilatação }\end{array}$ & $(18,24)$ \\
\hline $\begin{array}{l}\text { Poaceae, Cymbopogon citratus (DC.) Stapf., } \\
\text { Capim Limão }\end{array}$ & $\begin{array}{l}\text { Folha seca ou fresca } \\
\text { e raiz rizomatosa }\end{array}$ & $\begin{array}{l}\text { Redução da pressão arterial por um efeito direto } \\
\text { sobre o músculo liso vascular levando a vasodilatação }\end{array}$ & $(18,25)$ \\
\hline $\begin{array}{c}\text { Zingiberaceae, Sechium edule (Jacq.) Sw., } \\
\text { Chuchu }\end{array}$ & Flor, semente, raiz & Efeito vasorrelaxante & $(26,27)$ \\
\hline
\end{tabular}

Allium sativum L. (Alho):

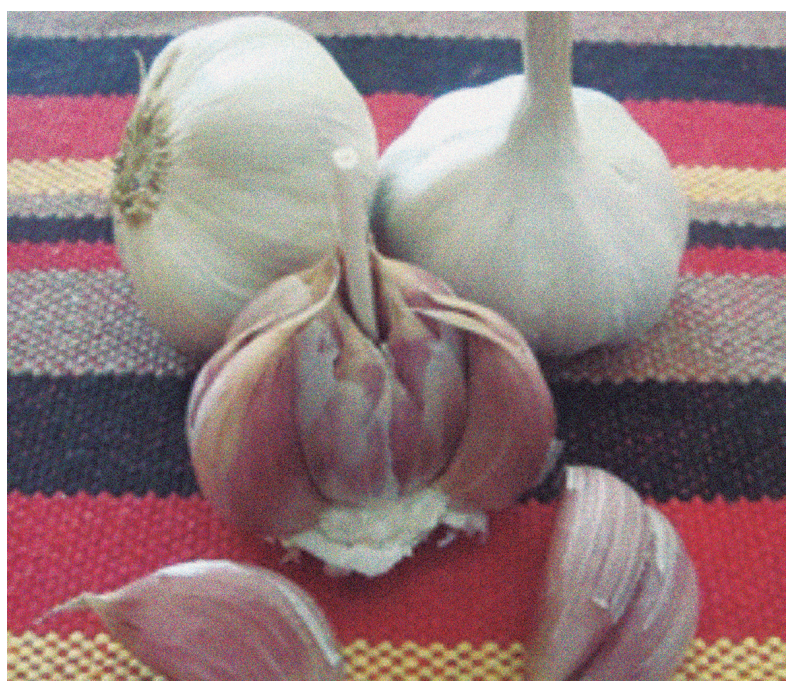

Constituintes: Esta planta apresenta em sua composição compostos sulfurados, aliina, alicina e ajoeno, além de glicosídeos, monoterpenoides, enzimas, vitaminas, minerais, flavonoides (campferol e quercetina) e saponinas (28).

Mecanismo de ação: Os compostos sulfurados mostraram atividade vasodilatadora in vitro mediada por liberação de óxido nítrico (29).

O uso do alho pode minimizar a expressão das isoformas CYP3A4, CYP3A5, CYP3A7, CYP2C9, CYP2C19 e CYP2E1 e da glicoproteína-P. Pode ainda causar um aumento da expressão da CYP2C9, CYP3A1, CYP1A1 (29).
Interações medicamentosas: Podem ocorrer interações sinérgicas quando o alho for usado simultaneamente ao fármaco nevibolol, um anti-hipertensivo $\beta$-bloqueador. Este medicamento atua inicialmente diminuindo o débito cardíaco. Provoca diminuição da secreção de renina, causa readaptação de barorreceptores e diminuição das catecolaminas nas sinapses nervosas. Atua ainda proporcionando vasodilatação pelo aumento da síntese e liberação endotelial de óxido nítrico (30), sendo este um efeito semelhante ao dos compostos sulfurados presentes no alho, sugerindo assim uma potencialização do efeito.

Foram descritas na literatura interações com os anti-hipertensivos inibidores da enzima conversora da angiotensina (ECA) - lisinopril - causando um aumento do efeito hipotensor do medicamento, e também com anticoagulantes orais, hipoglicemiantes, hipertiroideanos, anti-inflamatórios não esteroidais, antiviral (saquinavir), relaxante muscular (clorzoxazona), que são fármacos metabolizados pelo sistema enzimático P450 (28-29, 31-32).

Os antagonistas dos canais de cálcio sofrem metabolização no CYP450, sendo o verapamil metabolizado principalmente pela isoforma CYP3A4. Os $\beta$-bloqueadores (propranolol) são metabolizados pela isoforma CYP2D6, e sofrem interferência na presença do alho, sugerindo que ocorram interações farmacocinéticas, quando utilizados concomitante ao uso planta. (33). 
Alpinia zerumbet (Colônia)

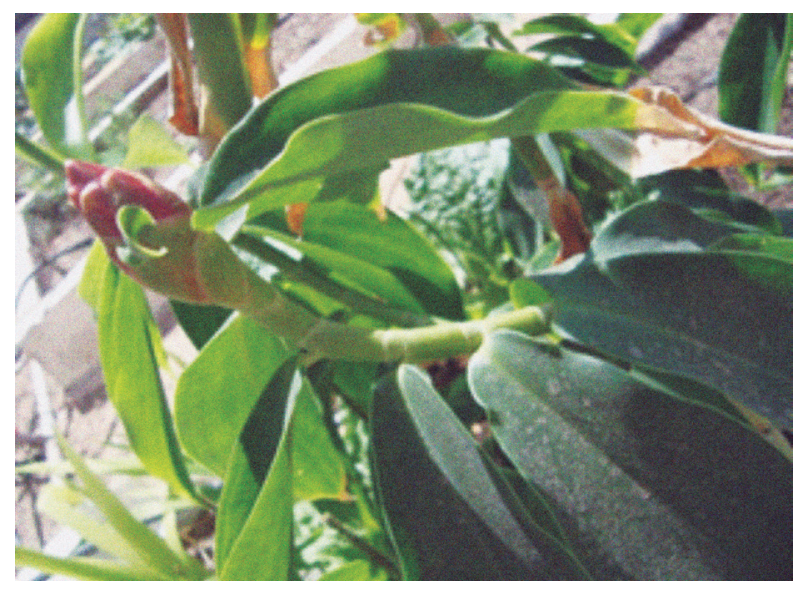

Constituintes: O óleo essencial é rico em mono- e sesquiterpenos, com maior concentração de terpinen-4-ol e 1,8-cineol, compostos responsáveis pela ação hipotensora e pela vasodilatação, respectivamente. Contudo entre seus componentes fixos, os mais importantes são os flavonoides e as kavapironas. A presença de flavonoides contribui para a ação anti-hipertensiva da planta (34-36).

Mecanismo de ação: Segundo Barcelos e cols (2010) o tratamento crônico com óleo essencial de $A$. zerumbet foi capaz de produzir redução da pressão arterial média e da hipertrofia cardíaca, no modelo de hipertensão experimental em ratos espontaneamente hipertensos, provavelmente pela presença dos componentes terpinen-4-ol e 1,8-cineol, o que poderia determinar melhoria da hemodinâmica cardiovascular dos animais hipertensos tratados (34). O terpineol atua bloqueando o influxo de cálcio nos canais, já as catequinas atuam diretamente na musculatura lisa vascular com ação diurética (37).

A administração de cápsulas de folhas secas pulverizadas de $A$. zerumbet em pacientes com hipertensão leve e moderada resultou em atividade anti-hipertensiva e vasodilatadora, a qual foi atribuída à presença de flavonoides no extrato foliar. Já o efeito anti-hipertensivo do extrato hidroalcoólico foi decorrente da vasodilatação e consequente diminuição da resistência vascular periférica (36).

Interações medicamentosas: $\mathrm{O}$ terpineol presente na planta atua de forma semelhante aos anti-hipertensivos antagonistas do cálcio (verapamil, nifedipino, anlodipina, diltiazem) que agem impedindo a entrada de cálcio nas células. $\mathrm{O}$ uso de $A$. zerumbet juntamente com medicamentos dessa classe pode causar hipotensão por aumentar o efeito anti-hipertensivo. As catequinas apre- sentam ação farmacológica semelhante ao mecanismo de ação dos anti-hipertensivos vasodilatadores diretos (hidralazina e minoxidil) $(30,38)$ podendo potencializar a ação desses fármacos quando usados em associação a planta.

\section{Citrus sp. (Laranja)}

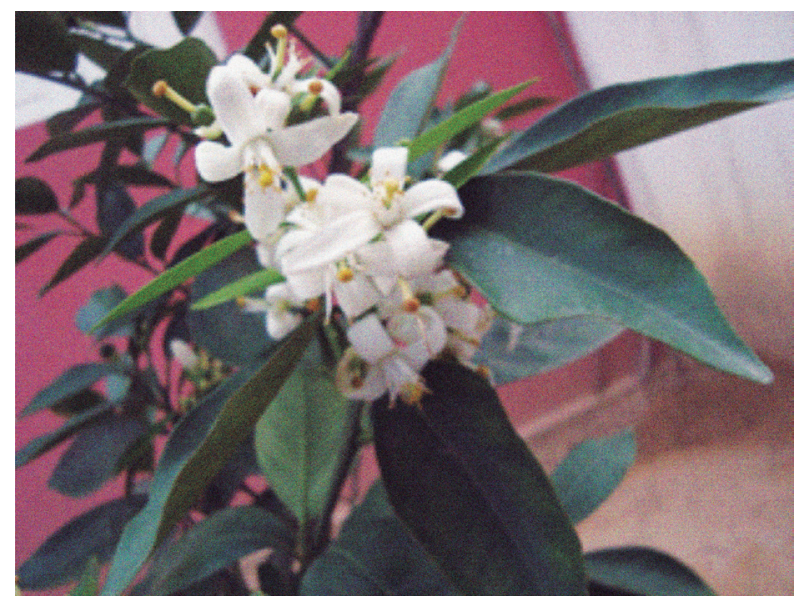

Constituintes: Fenóis, taninos, flavonoides (antocianidinas, flavanonas, flavonóis), hesperidina, xantonas, saponinas, esteroides e triterpenoides (39).

De acordo com Bonifácio e César (2009), a hesperidina presente em sucos e frutas cítricas possui atividade hipotensora em ratos normais e hipertensos e apresenta ação diurética em ratos normais. Contribui ainda com efeitos $\beta$-bloqueadores, culminando em efeito anti-hipertensivo e inibindo a atividade da ECA (40).

Mecanismo de ação: A hesperidina presente na planta bloqueia receptores $\beta$-adrenérgicos, assim como ocorre com fármacos que atuam na redução do débito cardíaco, reduz a secreção de renina pelas células justaglomerulares e possui ação central diminuindo a atividade simpática (41).

Interações medicamentosas: Segundo Lima e cols (2012) o consumo de suco de laranja vermelha por um período de oito semanas provocou uma redução da pressão arterial sistólica em pessoas eutróficas, e as pessoas com excesso de peso tiveram uma diminuição da pressão diastólica (23). Possivelmente ocorrem interações do tipo sinérgicas com fármacos anti-hipertensivos $\beta$-bloqueadores (atenolol, bisaprolol, metoprolol, propranolol, pindolol, caverdilol, nadolol e labetalol) (41).

Os inibidores da ECA (benazepril, captopril, cilazapril, delapril, enalapril, fosinopril, 1sinopril, perindopril, quinapril, ramipril, trandolapril) operam essencialmente por inibição da enzima conversora de angiotensina, blo- 


\section{Ungarma}

queando a enzima que converte angiotensina I em angiotensina II no sangue e tecidos (42). Tendo em vista que o mecanismo de ação de Citrus sp. atua de forma semelhante, inibindo a atividade dessa enzima, pode ser sugerido que ocorra uma interação aditiva à atividade do fármaco quando utilizados simultaneamente.

\section{Passiflora sp. (Maracujá)}

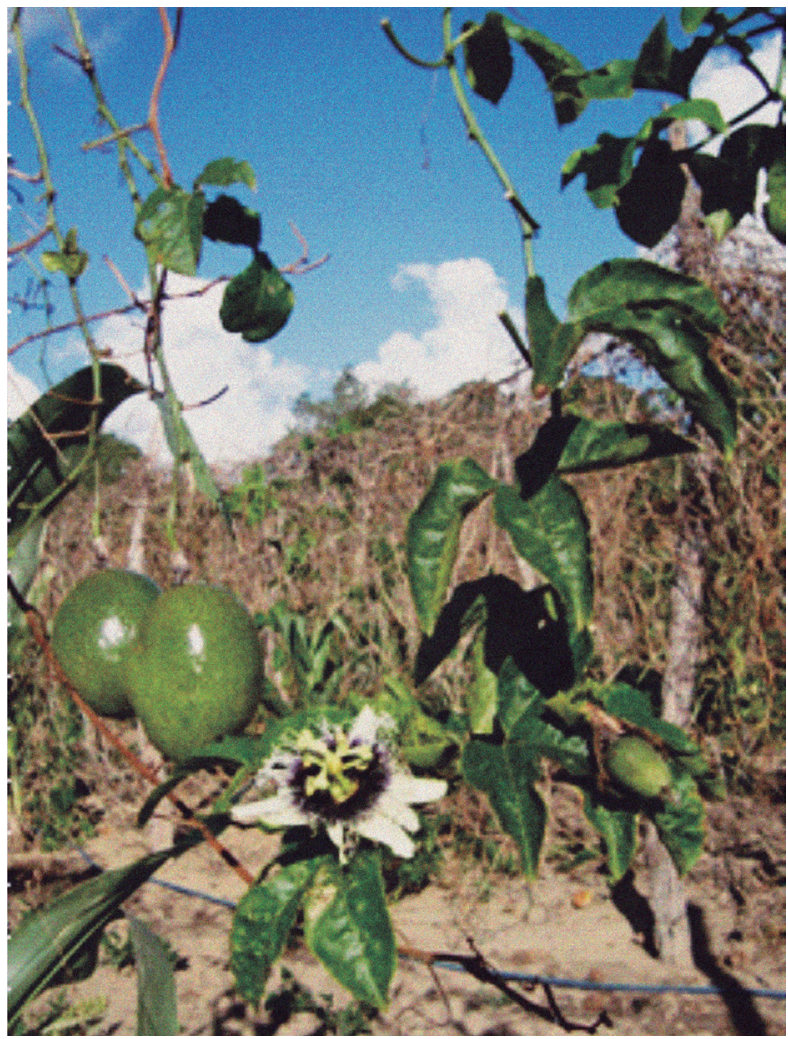

Constituintes: Os constituintes químicos mais frequentes citados para as espécies são os flavonoides, alcaloides indólicos e saponinas $(15,43)$.

Mecanismo de ação: Foi observado por Ichimura e cols (2006) a diminuição da pressão arterial sistólica em ratos espontaneamente hipertensos após administração de extrato metanólico de Passiflora edulis (10-15 mg/kg). Os autores sugerem que possivelmente o efeito esteja relacionado com a ação vasodilatadora de polifenóis como a luteolina e seus glicosídeos contidos no extrato (44).

Konta e cols (2013) avaliaram a atividade hipotensora da polpa do maracujá em ratos espontaneamente hipertensos. Os estudos revelaram que a mesma diminuiu de forma expressiva a pressão arterial sistólica. Esse efeito foi atribuído às propriedades antioxidantes dos compostos fenólicos que aprimoram a função endotelial e normatiza o tônus vascular resultando em efeito hipotensor (20).

Interações medicamentosas: o uso de Passiflora sp. associado à cafeína, guaraná ou efedra pode acarretar em aumento da pressão arterial. Os anti-hipertensivos da classe dos vasodilatadores diretos (hidralazina, minoxidil) atuam promovendo relaxamento da musculatura da parede vascular, resultando em vasodilatação e diminuição da resistência vascular periférica. Estes, quando associados ao uso de Passiflora sp podem potencializar o efeito farmacológico, visto que, o mecanismo de ação pelo qual a planta atua é semelhante (45).

\section{Lippia alba (Erva cidreira, Lípia)}

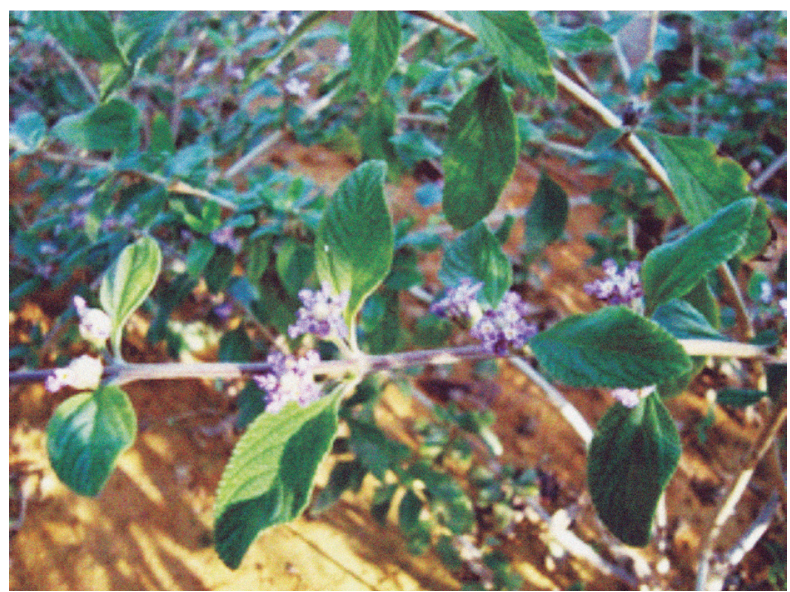

Constituintes: $\mathrm{O}$ óleo essencial é constituído por citronelol, citral, mirceno, limoneno e carvona (35).

Mecanismo de ação: $O$ citronelol é o responsável pela atividade anti-hipertensiva da planta. Estudos realizados por Bastos (2009) sugeriram que o mecanismo pelo qual o citronelol diminui a pressão arterial seja por efeito direto na musculatura lisa vascular, promovendo vasodilatação (24).

Interações medicamentosas: $\mathrm{O}$ citronelol presente em $L$. alba possui mecanismo de ação anti-hipertensivo semelhante aos vasodilatadores diretos (hidralazina, minoxidil, nitroprussiato) que agem sobre a musculatura da parede vascular, proporcionando relaxamento muscular, vasodilatação e a diminuição da resistência vascular periférica (30). Essa semelhança no mecanismo de ação sugere que a associação entre essa classe de fármacos e a Lippia alba pode causar uma potencialização do efeito destes fármacos. 


\section{Cymbopogon citratus (Capim Limão)}

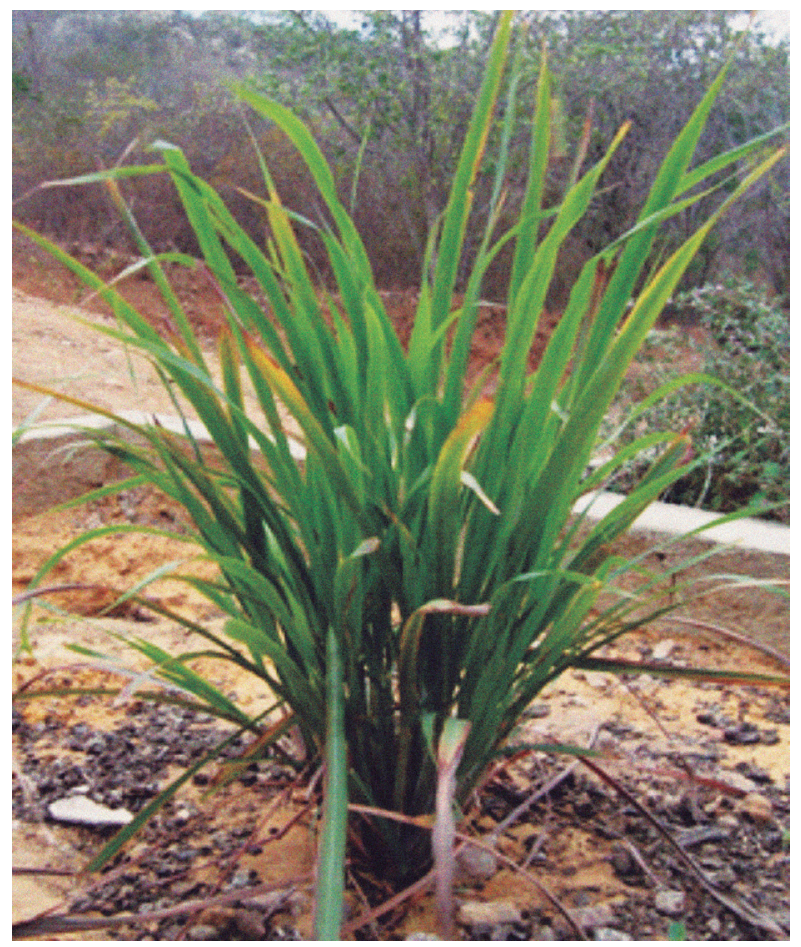

Constituintes: Os principais constituintes do óleo essencial do Cymbopogon citratus descritos no estudo de Carmo e cols (2012) foram geraniol, neral e mirceno (46).

Mecanismo de ação: $O$ óleo essencial provocou hipotensão, presumivelmente por causa da redução da resistência vascular resultante da inibição do influxo de $\mathrm{Ca}^{2+}$ e possivelmente por ativação de receptores muscarínicos cardíacos que provocam a bradicardia (9).

Interações medicamentosas: Podem ocorrer interações sinérgicas dos antagonistas dos canais de cálcio, uma vez que o mecanismo pelo qual os constituintes do óleo essencial de $C$. citratus coincide com o mecanismo de ação dos fármacos que estão em três classes diferentes: fenilalquilaminas (verapamil), benzotiazepinas (diltiazem) e diidropiridinas (nifedipina e anlodipina). Essas diferentes classes ligam-se as subunidades $\alpha_{1}$ dos canais de cálcio do tipo $\mathrm{L}$, impedindo a abertura dos canais de cálcio. Os efeitos no músculo liso são a dilatação arterial generalizada e a redução da resistência arteriolar, com consequente diminuição da pressão $(38,23)$.

\section{Sechium edule (Chuchu)}

Constituintes: Os principais constituintes presentes na planta que são responsáveis por sua atividade farmacológica são as saponinas e os flavonoides (32).

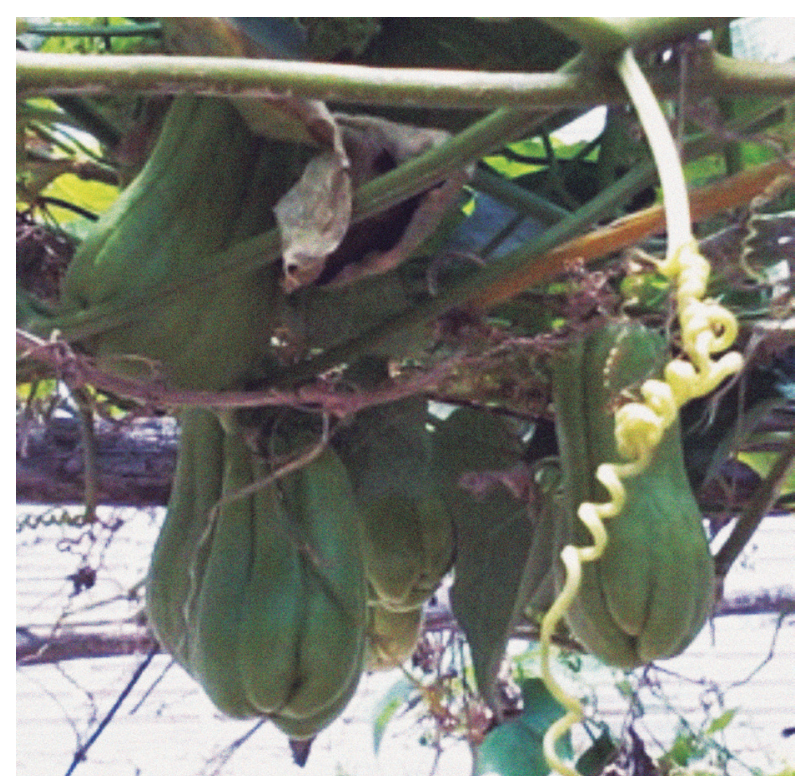

Mecanismo de ação: Lombardo-earl e cols (2014) comprovaram a atividade anti-hipertensiva do chuchu utilizando o extrato hidroalcoólico da raiz (27), corroborando com o uso popular das folhas e da polpa para obtenção de efeito hipotensor (26).

Interações medicamentosas: Segundo Maia e cols (2011), Sechium edule apresenta interações medicamentosas com hipotensores, diuréticos, sedativos, calmantes e anti-histamínicos (32).

Para além da capacidade de tratar pacientes com sucesso, os medicamentos têm potencial para provocar danos. Interações medicamentosas figuram entre os fatores responsáveis pelos efeitos nocivos causados pelos fármacos (14). Interações entre plantas medicinais e fármacos são baseadas nos mesmos mecanismos de farmacocinética e farmacodinâmica das interações fármaco-fármaco, podendo modificar a concentração plasmática do fármaco objeto e como consequência levar a níveis terapêuticos tóxicos ou subdoses dependendo da inibição ou indução das enzimas que o metabolizam $(8,47)$.

As informações obtidas ao longo da revisão de literatura evidenciaram que as principais interações envolvidas no uso de plantas medicinais associadas a fármacos para tratar a hipertensão arterial são, em sua maioria, de natureza farmacodinâmica causando efeito aditivo ou sinérgico.

A maioria dos medicamentos, preparações à base de plantas e nutracêuticos apresentam efeitos notáveis sobre vias bioquímicas e podem influenciar na função cardiovascular, dentre outros processos biológicos. Podem também interagir com outros medicamentos prescritos (48). 
Saad e cols (2016) afirmaram que as ações farmacológicas das plantas com atividade anti-hipertensiva (diurética, sedativa, inibidora da ECA, inibidora da ação do canal de cálcio) são semelhantes às das substâncias sintéticas usadas nessas situações. Porém, aa interação sinérgica que ocorre entre os vários constituintes do fito- complexo resulta em ação mais suave com menos efeitos colaterais (15).

O Quadro 2 ilustra as possibilidades de interação das plantas descritas com as classes de medicamentos anti-hipertensivos utilizados na terapia convencional.

Quadro 2. Interações de plantas medicinais com medicamentos anti-hipertensivos.

\begin{tabular}{|c|c|c|c|c|c|c|c|}
\hline \multirow{2}{*}{$\begin{array}{l}\text { CLASSE DE MEDICAMENTOS } \\
\text { ANTI-HIPERTENSIVO }\end{array}$} & \multicolumn{7}{|c|}{ PLANTA } \\
\hline & Alho $^{1}$ & Colônia $^{2}$ & Maracujá ${ }^{3}$ & Laranja $^{4}$ & $\begin{array}{c}\text { Erva } \\
\text { Cidreira }^{5}\end{array}$ & $\begin{array}{l}\text { Capim } \\
\text { Limão }^{6}\end{array}$ & Chuchu $^{7}$ \\
\hline \multicolumn{8}{|l|}{ Diuréticos (Furosemida) } \\
\hline \multicolumn{8}{|l|}{$\begin{array}{l}\text { Antagonistas-adrenérgicos } \\
\text { (Propranolol) }\end{array}$} \\
\hline \multicolumn{8}{|l|}{ IECA (Captopril) } \\
\hline \multicolumn{8}{|l|}{$\begin{array}{l}\text { Bloqueadores dos canais de cálcio } \\
\text { (Anlodipino) }\end{array}$} \\
\hline $\begin{array}{l}\text { Vasodilatadores diretos } \\
\text { (Hidralazina) }\end{array}$ & & & & & & & \\
\hline
\end{tabular}

${ }^{1}$ Allium sativum, ${ }^{2}$ Alpinia zerumbet, ${ }^{3}$ Passiflora sp., ${ }^{4}$ Citrus sp., ${ }^{5}$ Lippia alba, ${ }^{6}$ Cymbopogon citratus, ${ }^{7}$ Sechium edule.

Não foram encontrados, na literatura, relatos de interação das plantas investigadas com fármacos pertencentes aos grupos dos simpatolílicos, antagonistas dos receptores AT1 de angiotensina II e inibidores da endotelina.

Com relação às interações do tipo farmacocinética, foi observado efeito apenas com o uso do alho associado aos anti-hipertensivos, pois o mesmo inibe algumas enzimas hepáticas causando acúmulo de medicamento no organismo, ou ainda pode aumentar a expressão de algumas enzimas hepáticas, excretando o fármaco rapidamente.

A modulação das enzimas hepáticas (CYP e glicoproteína-P) por plantas medicinais pode ter implicações clínicas e toxicológicas significativas, sendo necessária avaliação rigorosa para a possibilidade de interações de planta-fármaco no processo de desenvolvimento de fitoterápicos (49).

É relevante considerar que fatores farmacocinéticos se alteram com o envelhecimento, e que doses de medicamentos muitas vezes precisam de ajustes para evitar eventos adversos (49). Como o número de indivíduos, particularmente os idosos, que utilizam produtos à base de plantas junto com medicamentos convencionais continua a crescer, o risco de interações aumenta, mas ainda é pouco reconhecido (48).

A verdadeira incidência e a natureza das reações adversas de plantas medicinais ou interações com fár- macos permanecem desconhecidas, dada a dificuldade de vigilância pós-comercialização. Os eventos adversos são subnotificados e, além disso, as informações sobre a segurança são frequentemente baseadas em relatos de casos e estudos realizados em um número limitado de jovens voluntários saudáveis ou pacientes com comorbidades limitadas. Portanto, falta evidência para a segurança dos produtos à base de plantas em idosos, e a verdadeira magnitude dos problemas que as interações de planta-fármaco representam para a saúde pública, em particular, para pacientes idosos com doenças cardiovasculares (48).

Apesar do volume de pesquisas, a segurança e a efetividade da medicina alternative e complementar permanecem largamente desconhecidas, mas apresentam fortes evidências no uso tradicional, sendo esta reconhecida como parte da comprovação da eficácia e segurança de produtos naturais, conforme critérios estabelecidos na RDC 14/2010 da ANVISA (50). No entanto, o efeito adverso da própria planta, a adulteração, a formulação inadequada, a falta de compreensão das interações de plantas e medicamentos sintéticos podem levar a reações adversas. Somando-se a isso, os padrões de controle de qualidade são altamente variáveis, bem como a comercialização de muitos produtos enganosos ou potencialmente prejudiciais quando usados pela população vulnerável. Para minimizar o dano, são necessários me- 
dicamentos de boa qualidade, com segurança e eficácia, e utilizados de forma racional $(8,48)$

Melhorias contínuas na compreensão sobre as interações planta-fármaco e seus mecanismos farmacocinéticos nos permitirão prever melhor, avaliar e gerir os riscos potenciais associados às terapias baseadas no uso concomitante de plantas medicinais e fármacos sintéticos (51).

Considerando que doenças cardiovasculares são de elevado risco de vida, exigindo o uso de múltiplos medicamentos, e que estas ocorrem em uma população com comorbidades extensas, o risco de interações planta-fármaco não pode ser ignorado (48). $\mathrm{O}$ risco de dano, no entanto, é menor quando os medicamentos usados são acompanhados por profissionais de saúde informados e por pacientes que entendam a responsabilidade do uso de seus medicamentos à base de plantas. Esforços para facilitar a comunicação entre pacientes e médicos a respeito de um risco clínico de interações medicamentosas de plantas medicinais também devem ser incentivados, pois o uso supervisionado, associado ao tratamento convencional tornam os efeitos colaterais e as interações, raras. Além disso, os profissionais de saúde representam um papel crucial na disseminação do uso racional de medicamentos, porque as atitudes adotadas por eles ganham legitimidade perante a população $(7,51,52)$.

Pode ser percebido que o uso de plantas medicinais no tratamento de doenças crônicas como a hipertensão arterial é uma estratégia bastante promissora e cada vez mais estudada pela comunidade científica. Porém, o uso dessa terapia deve ser feita com cautela, pois mesmo existindo estudos científicos que comprovem a atividade farmacológica das plantas, alguns mecanismos pelos quais produzem seus efeitos ainda não são completamente elucidados, assim como os componentes envolvidos na atividade farmacológica.

No entanto, há que ser considerado que as interações podem ocorrer por meio da utilização das plantas medicinais em práticas de automedicação. Nesses casos, dificilmente o médico é informado, o que pode trazer efeitos adversos e intoxicantes, alterando os resultados desejados dos medicamentos convencionais e fitoterápi$\cos (9)$.

Segundo Martins e cols (2016), em um estudo de investigação da relação entre o uso de plantas medicinais e a adesão medicamentosa por idosos, o uso de plantas medicinais é frequentemente associado pelo indivíduo como algo que está fora da preocupação do profissional de saúde seja por medo da desaprovação, ou porque o paciente pensa que é irrelevante informar, por ser desprovido de efeitos colaterais, ou interações como é frequente, e erroneamente assumido (53).

Em suma, os estudos disponíveis mostram potenciais interações entre plantas medicinais e medicamentos convencionais utilizados no tratamento de hipertensão. As informações obtidas ao longo do presente trabalho forneceram bases para alertar os profissionais da saúde para a necessidade do reconhecimento dos riscos de interação, bem como da importância de um melhor acompanhamento, buscando evitar danos à saúde do paciente e oferecendo maiores informações para conscientização do uso racional das plantas medicinais.

As interações mais comuns envolvidas no uso de plantas medicinais associadas ao uso de fármacos para tratar a hipertensão arterial foram do tipo farmacodinâmica com efeitos sinérgicos, seguidas de interações do tipo farmacocinética, por interferência nas enzimas hepáticas.

As plantas que mais apresentaram interações com os medicamentos convencionais foram o alho, seguido da colônia e laranja. E as classes farmacológicas anti-hipertensivas mais envolvidas foram os antagonistas dos canais de cálcio e os vasodilatadores diretos.

\section{CONCLUSÃO}

Neste contexto, se torna imprescindível que os profissionais da área da saúde fiquem atentos e questionem seus pacientes quanto ao uso concomitante de plantas medicinais e seus possíveis sintomas que evidenciem interações com medicamentos. É importante também que os usuários, no caso de utilizar medivcamentos convencionais juntamente com fitoterápicos ou plantas medicinais, solicitem informações aos profissionais da área da saúde, para evitar riscos de interação medicamentosa e prejudicar o seu tratamento.

Ressalta-se que os portadores de hipertensão em tratamento não devem utilizar plantas medicinais como tratamento auxiliar, sem orientação de profissional capacitado, pois estas podem causar interações com os fármacos, levando ao risco, principalmente, de quadros de hipotensão devido aos efeitos sinérgicos, bem como de insegurança, por potencializar os efeitos colaterais dos medicamentos. 


\section{REFERÊNCIAS}

1. Veras RP. Estratégias para o enfrentamento das doenças crônicas: um modelo em que todos ganham. Rev Bras de Geriat e Gerontol., 2011; 14(4): 779-786.

2. Malta DC, Oliveira MR, Moura EC, Silva AS, Zouain CS, Santos FP, Morais Neto OL, Penna GO. Fatores de risco e proteção para doenças crônicas não transmissíveis entre beneficiários da saúde suplementar: resultados do inquérito telefônico Vigitel, Brasil, 2008. Ciênc. Saúde Colet. 2011; 16(3): 2011-2022.

3. Rosário TM, Scala LCM, França GVA, Pereira MRG, Jardim PCB. Prevalence, control and treatment of arterial hypertension in Nobres-MT. Arq Bras Cardiol., 2009; 93(6): 622-628. DOI: 10.1590/S0066782X2009001200018.

4. Ferreira SRG, Moura EC, Malta, DC, Sarno F. Freqüência de hipertensão arterial e fatores associados: Brasil, 2006. Rev Saúde Públ., 2009; 43(2): 98-106.

5. Ferreira JS, Aydos RD. Prevalência de hipertensão arterial em crianças e adolescentes obesos. Ciênc Saúde Colet. 2010. 15(1):97-104. DOI: 10.1590/S141381232010000100015 .

6. Cock IE. The safe usage of herbal medicines: counter-indications, cross-reactivity and toxicity. Pharmacogn. Commun. 2015. 5(1):2.

7. Taniya T, Nardev S. A review on sideffects of herbal drugs. World. J. Pharm. Sci. 2016; 5(6):2150-2164. DOI: 10.20959/wjpps20166-6983.

8. Mamindla S, Prasad KVSRG, Koganti B. Herb-drug interactions: an overview of mechanisms and clinical aspects. Int. J. Pharm. Sci. Res. 2016; 7(9): 3576. DOI: 10.13040/IJPSR.0975-8232.7(9).3576-86.

9. Moreira FV, Bastos JF, Blank AF, Alves PB, Santos MR. Chemical composition and cardiovascular effects induced by the essential oil of Cymbopogon citratus DC. Stapf, Poaceae, in rats. Rev. Bras. Farmacogn. 2010; 20(6):904-909.

10. Moreira TMS, Salgado HRN, Pietro RCLR. O Brasil no contexto de controle de qualidade de plantas medicinais. Rev Bras Farmacog. 2010; 435-440. DOI: 10.1590/ S0102-695X2010000300023.

11. Amorim MFD, Diniz MFFM, Araújo MST, Pita JCLR, Dantas JG, Ramalho JA, Xavier AL, Palomaro TV, B-Júnior NL. The controvertible role of kava (Piper methysticum G. Foster) an anxiolytic herb, on toxic hepatites. Rev. Bras. Farmacogn. 2007; 17(3): 448-454. DOI: 10.1590/S0102-695X2007000300020.

12. Veiga Junior VF. Estudo do consumo de plantas medicinais na Região Centro-Norte do Estado do Rio de Janeiro: aceitação pelos profissionais de saúde e modo de uso pela população. Rev. Bras. Farmacogn. 2008; 18(2): 308-313. DOI: 10.1590/S0102-695X2008000200027.

13. Alexandre RF, Bagatini F, Simões CMO. Interações entre fármacos e medicamentos fitoterápicos à base de ginkgo ou ginseng. Rev. Bras. Farmacogn. 2008; 18(1):117-126. DOI: 10.1590/S0102-695X2008000100021.

14. Abubakar AR, Chedi BA, Mohammed KG, Haque M. Drug interaction and its implication in clinical practice and personalized medicine. Nat J Physiol, Pharm Pharmacol. 2015; 5(5):343-349. DOI: 10.5455/ njppp.2015.5.2005201557.

15. Saad GDA, Léda PHO, Sá IM, Seixlack ACC. Fitoterapia contemporânea: tradição e ciência na prática clínica. 2. ed. Rio de Janeiro: Guanabara Koogan, 2016.

16. Mendes KDS, Silveira RCC, Galvão CM. Revisão integrativa: método de pesquisa para a incorporação de evidências na saúde e na enfermagem. Texto Contexto Enferm. 2008; 17(4): 758-764. DOI: 10.1590/S010407072008000400018.

17. Gil AC. Como elaborar projetos de pesquisa. 4. ed. São Paulo: Atlas, 2009.

18. Oliveira FCS, Barros RFM, Moita Neto JM. Plantas medicinais utilizadas em comunidades rurais de Oeiras, semiárido piauiense. Rev Bras Plantas Med. 2010; 12(3): 282-301. DOI: 10.1590/S1516-05722010000300006.

19. Nunes MGS, Bernardino AO, Martins RD. Uso de plantas medicinais por pessoas com hipertensão. Rev Rene. 2015; 16(6):775-781.

20. Konta EM, Almeida MR, Amaral CL, Darin JD, Rosso VV, Mercadante AZ, Antunes LM, Bianchi ML. Evaluation of the Antihypertensive Properties of Yellow Passion Fruit Pulp (Passiflora edulis Sims f. flavicarpa Deg.) in Spontaneously Hypertensive Rats. Phytother Res. 2013; 28(1): 28-32. DOI: 10.1002/ptr.4949.

21. Ribeiro DA, Macêdo DG, Oliveira LGS, Saraiva ME, Oliveira SF, Souza MMA, Menezes IRA. Potencial terapêutico e uso de plantas medicinais em uma área de Caatinga no estado do Ceará, Nordeste do Brasil. Rev Bras Plant Med. 2014; 16(4): 912-930. DOI: 10.1590/1983084X/13 059.

22. Borges KN, Bautista H. Etnobotânica de Plantas Medicinais na Comunidade de Cordoaria, Litoral Norte do Estado da Bahia, Brasil. Plurais-Rev. Multidisc. 2010; 1(2):153-174.

23. Lima CG, Basile LG, Silveira JQ, Cesar TB. Ingestão regular do suco de laranja vermelha reduz pressão arterial de adultos. J Health Sci Inst., 2012; 30(1): 59-63.

24. Bastos JFA. Hypotensive and Vasorelaxant Effects of Citronellol, a Monoterpene Alcohol, in Rats. Basic Clin Pharmacol. Toxicol. 2009; 106: 331-337.

25. Lopes GAD, Feliciano LM, Diniz REDS, Alves MJQDF. Plantas medicinais: indicação popular de uso no tratamento de hipertensão arterial sistêmica (HAS). Rev. Ciênc. Exten. 2010; 6(2):143-155.

26. Costa VP, Mayworm MAS. Plantas medicinais utilizadas pela comunidade do bairro dos Tenentes - município de 
Extrema, MG, Brasil. Rev Bras Plantas Med. 2011; 13(3): 282-292. DOI: 10.1590/S1516-05722011000300006.

27. Lombardo-Earl G, Ramos RR, Zamilpa A, Ruiz MH, Salgado GR, Tortoriello J, Ferrer EJ. Extracts and Fractions from Edible Roots of Sechium edule (Jacq.) Sw. with Antihypertensive Activity. Evid Based Complement Altern Med. 2014; 1: 1-9. DOI: 10.1155/2014/594326.

28. Williamson E, Driver S, Baxter K. Interações Medicamentosas de Stockley. Porto Alegre: Artmed, 2012.

29. Alexandre RF, Bagatini F, Simões CMO. Potenciais interações entre fármacos e produtos à base de valeriana ou alho. Rev. Bras. Farmacogn.. 2008; 18: 455-463. DOI: 10.1590/S0102-695X2008000300021.

30. Kohlmann Jr O, Gus M, Ribeiro AB, Vianna D, Coelho EB, Barbosa E, Almeida FA, Feitosa G, Moreno H, Guimarães I, Ribeiro JP, Ramirez JAF, Martins JFV, Santos RAS. Tratamento medicamentoso. J. Bras. Nefrol. Supl. 2010; 32(1): 29-43. DOI: 10.1590/S010128002010000500008.

31. Santiago MB. Efeito da administração do Allium sativum sobre as alterações cardiovasculares de ratos Wistar com infarto do miocárdio. Rev Ciênc Farm Básica Apl. 2009; 30(1):62-69.

32. Maia LF, Castro QJT, Resende FMF, Rodrigues-das-Dores RG. Plantas medicinais e hipertensão. Pharm Rev. $2011 ; 24: 24-25$.

33. Silva BQ, Hahn SR. Uso de plantas medicinais por indivíduos com hipertensão arterial sistêmica, diabetes mellitus ou dislipidemias. Rev Bras Farm Hospitalar e Serv de Saúde. 2011; 2(3): 36-40.

34. Barcelos FF, Oliveira ML, Giovaninni NPB, Lins TP, Filomeno CA, Schneider SZ, Pinto VD, Endringer DC, Andrade TU. Estudo químico e da atividade biológica cardiovascular do óleo essencial de folhas de Alpinia zerumbet (Pers.) B.L.Burtt \& R.M.Sm. em ratos. Rev. Bras. Plantas Med. 2010; 12(1):48-56. DOI: 10.1590/ S1516-05722010000100008.

35. Oliveira GL, Oliveira AFM, Andrade LHC. Plantas medicinais utilizadas na comunidade urbana de Muribeca, Nordeste do Brasil. Acta Bot Bras. 2010; 24(2): 571-577. DOI: $10.1590 / \mathrm{S} 0102-33062010000200026$.

36. Gorzalczany S, Moscatelli V, Ferraro G. Artemisia copa aqueous extract as vasorelaxant and hypotensive. J. Ethopharmacol. 2013; 148(1): 56-61.

37. RJ. Prefeitura Municipal do Rio de Janeiro. Manual Terapêutico de Fitoterápicos: Programa de Plantas Medicinais, 25 p., 2010.

38. Rang, HP, Dale MM, Ritter JM, Flower RJ, Henderson G. Farmacologia. $7^{\mathrm{a}}$ ed. Rio de Janeiro: Elsevier, 2011.

39. Carlos LC, Mota EI, Silva FI, Souza AM, Bertini LM. Estudo fitoquímico dos extratos e frações obtidas a partir do extrato etanólico de Citrus limonium. In: VII CONNEPI-Congresso Norte Nordeste de Pesquisa e Inovação, 2012
40. Bonifácio NP, César TB. Influência da ingestão crônica do suco de laranja na pressão arterial e na composição corporal. Rev. Bras. Hipertens. 2009; 16(2): 76-81.

41. Longo MA, Martelli A, Zimmermann A. Hipertensão Arterial Sistêmica: aspectos clínicos e análise farmacológica no tratamento dos pacientes de um setor de Psicogeriatria do Instituto Bairral de Psiquiatria, no Município de Itapira, SP. Rev Bras Geriat Geontol. 2011; 14(2): 271-284.

42. SBC. Sociedade Brasileira de Cardiologia. Departamento de Hipertensão Arterial. VI Diretrizes Brasileiras de Hipertensão Arterial. Rev. Bras. Hipertens. 2010; 17(1): $1-66$.

43. Gosmann G, Provensi G, Comunello LN, Rates SMK. Composição química e aspectos farmacológicos de espécies de Passiflora L. (Passifloraceae). Rev Bras Bioci. 2011; 9(1): 88-99.

44. Ichimura T, Yamanaka A, Ichiba T, Toyokawa T, Kamada Y, Tamamura T, Maruyama S. Antihypertensive effect of an extract of Passiflora edulis rind in spontaneously hypertensive rats. Biosc, Biotech Biochem. 2007; 70(3): 718-721. DOI: 10.1271/bbb.70.718.

45. Nicoletti MA, Oliveira-Júnior MA, Bertasso CC, Caporossi PY, Tavares APL. Principais interações no uso de medicamentos fitoterápicos. Infarma. 2007; 19(1/2):3240.

46. Carmo ES, Pereira FO, Moreira ACP, Brito LL, Gayoso CW, Costa JGM, Lima EO. Essential oil from Cymbopogon citratus (DC) Stapf: a promising natural product against Malassezia spp. Rev. Inst. Adolfo Lutz. 2012; 71(2): 386-391.

47. Mouid MG. Effect of Ethanolic Extract of Aerial Parts of Andrographis paniculata on the Pharmacokinetics of Gliclazide in Rats. Asian J Biomed Pharm Scienc. 2015; 5(51): 21. DOI: 10.15272/ajbps.v5i51.755.

48. Sultan S, Viqar M, Ali R, Tajik AJ, Jahangir A. Essentials of herb-drug interactions in the elderly with cardiovascular disease. J. Patient.-Cent. Res. Rev. 2015; 2:174-191. DOI: $10.17294 / 2330-0698.1212$.

49. Davies EA, O'Mahony MS. Adverse drug reactions in special populations-the elderly. British J Clin Pharmacol. 2015; 80(4): 796-807.

50. BRASIL. . Dispõe sobre o registro de medicamentos fitoterápicos, RDC nº 14 de 30 de março de 2010.

51. Cho HJ, Yoon IS. Pharmacokinetic interactions of herbs with cytochrome p450 and p-glycoprotein. Evid.-Based Compl. Altern. Med. 2015; DOI: 10.1155/2015/736431.

52. Mukhtar O, Weinman J, Jackson SHD. Intentional non-adherence to medications by older adults. Drugs Aging. 2014; 31: 149-157.

53. Martins RR, Farias AD, Martins RR, Oliveira AG. Influence of the use of medicinal plants in medication adherence in elderly people. Int J Clin Pract. 2016; 70(3): 254-260. DOI: 10.1111/ijcp.12773. 